\title{
Bemerkungen zu der Arbeit von E. Grafe und V. Schläpfer:1) *Über Stickstoffretentionen und Stickstoffgleichgewicht bei Futterung von Ammoniaksalzen.»
}

\author{
Von \\ Wilhelm Völtz.
}

(Der Redaktion zugegangen am 13. Juni 1912.)

In dieser vor kurzem publizierten Arbeit, welche die Autoren übrigens zu denselben Ergebnissen führte, zu denen ich bereits vor längerer Zeit auf Grund eigener diesbezüglicher Untersuchungen gelangt bin, machen die Verfasser in mehreren wesentlichen Punkten unzutreffende Angaben über meine Versuchsanstellung und die daraus gezogenen Schlußfolgerungen. So wird von Grafe und Schläpfer hervorgehoben, daß ich mich vom praktischen Standpunkte aus mit der Frage befaßt hätte, ob bei den ‘Zuchttieren, (sämtlich Pflanzenfresser) Zugaben von Asparagin und Ammoniaksalzen einen günstigen Einfluß auf den Eiweißstoffwechsel ausüben. Diese Angabe entspricht nicht den Tatsachen. Meine diesbezüglichen Untersuchungen ") ${ }^{8}$ ) über die N-Retention nach Asparaginund Ammonacetatzufuhr sind an Fleischfressern (Hunden) zur Durchführung gelangt und hatten in erster Linie theoretisches Interesse. Es kam mir darauf an, festzustellen, wie groß der physiologische Nutzeffekt dieser und anderer einfach konstituierter N-haltiger Verbindungen ist, und ob die tierischen Zellen den Stickstoff der genannten Verbindungen eventuell zu verwerten vermögen. Diese Gesichtspunkte bestimmten mich dazu, Hunde als Versuchstiere zu wählen, weil die Verwertung der Nahrung beim Fleischfresser im Gegensatz zu Herbivoren nur wenig durch die Tätigkeit der Mikroorganismen im Verdauungstraktus kompliziert wird. Bei meinem ersten Versuch (1. c.) erhielt eine kleine, ca. 4,8 kg schwere ausgewachsene Hündin eine aus Fleisch, Reis und Schmalz bestehende

1) Diese Zeitschrift, Bd. 77, Heft 1, S. 1-21, 1912.

2) W. Völtz, Über das Verhalten einiger Amidsubstanzen allein und im Gemisch im Stoffwechsel der Karnivoren. Arch. für die ges. Physiologie, Bd. 112, S. 413-438, 1906.

s) W. Völtz (Referent) und G. Yakuwa, Über die Verwertung verschiedener Amidsubstanzen durch Karnivoren. Archiv für die ges. Physiologie, Bd. 121, S. 117-149, 1908. 
Grundration, zu der in den Hauptperioden als Zulage $1 \mathrm{~g}$ Stickstoff in Form der zu untersuchenden Amidsubstanzen (Asparagin, Ammonacetat, Acetamid, Glykokoll und endlich ein Gemisch der genannten Amide) gereicht wurde. Die Nahrung war sowohl hinsichtlich des Gehaltes an $\mathrm{N}$-haltigen Verbindungen als auch an $\mathrm{N}$-freien Stoffen reichlich bemessen, da pro Körperkilogramm und Tag rund $0,9 \mathrm{~g} \mathrm{~N}$ und $125 \mathrm{Kal}$., bezw. rund 110 nutzbare Kalorien verabreicht wurden. Infolge intensiverer und speziell $\mathrm{N}$-reicherer Ernährung waren bei der vorliegenden Versuchsreihe günstige Bedingungen für eine Stickstoffretention nicht vorhanden, nur während der 3 ersten Perioden wurden 0,5 g N (Grundfutterperiode) bezw. 0,29 g N (Asparaginperiode) bezw. 0,5 g N (Ammonacetatperiode) retiniert. Später bestand, mit Ausnahme der Amidgemischperiode (Ansatz 0,17 g N); nahezu Stickstoffgleichgewicht, und es trat insofern eine Komplikation ein, als sich im weiteren Verlauf des Versuches bei dem Versuchstier ein Darmkatarrh ausbildete, der zu einer kontinuierlich zunehmenden Stickstoffausscheidung in den Faeces führte.

In einer zweiten experimentellen Arbeit wurden von mir in Gemeinschaft mit G. Yakuwa (1. c. 3) an 3 Hündinnen 3 Versuchsreihen mit den genannten Amidsubstanzen durchgeführt, von denen die erste ${ }^{1}$ ) wegen Erkrankung des Versuchstieres vorzeitig abgebrochen werden mußte.

An dem zweiten Tier, und zwar einer wachsenden Hündin, wurden 12 Perioden von zumeist je 5 tägiger Dauer durchgeführt. Das Regime bestand auch hier aus Fleisch, Reis und Schmalz und während der Hauptperiode außerdem aus einer Zulage von $1 \mathrm{~g} \mathrm{~N}$ in Form der zu untersuchenden Amidstoffe. Jede Hauptperiode wurde von Grundrationsperioden eingeschlossen. Um günstige Bedingungen für Stickstoffretentionen zu schaffengirwar die Stickstoff- und Energiezufuhr bei der wachsenden Hündin $\mathrm{N}^{\prime} \mathrm{r} 2 \mathrm{we}=$ sentlich geringer bemessen als bei den Versuchen an den andere $v^{r}$ Tieren. Es wurden nämlich pro Körperkilogramm und Tag nur $0,4-0,6 ; \mathrm{N}$ und 80-90 Kalorien verabfolgt. Der Harn wurde täglich morgens: vor der Fütterung mittels Katheters abgegrenzt, sodaß man den Verlauf dir Kurve für den N-Gehalt des Urins in jeder Periode genau verfolgen kann. Uns interessiert zunächst Versuch mit der dem Ammonacetat. Die diesbezüglichen analytischen Daten lasse ich folgen:

Grundrationsperiode (27. Mai bis 1. Juni 1907)

Das Tier Nr. 2 (wachsende Hünỏin) erhielt pro die:

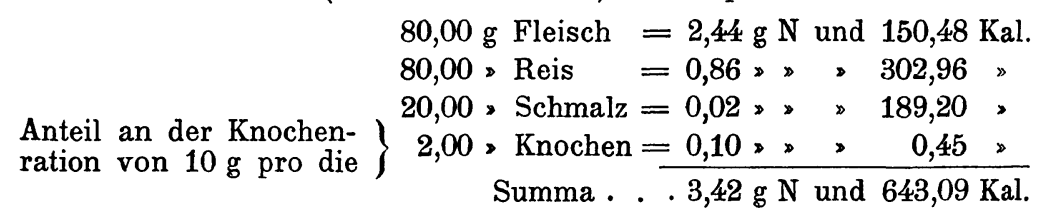

1) Es handelt sich um die in dem früheren Versuch (1. c. Anm. 2) von mir benutzte Hündin. 
Bemerkungen zu der Arbeit von E. Grafe und V. Schläpfer. 417

N-Bilanzen:

\begin{tabular}{|c|c|c|c|c|c|c|c|c|}
\hline \multirow[b]{2}{*}{$\begin{array}{l}\text { Datum } \\
1907 \\
\end{array}$} & \multirow{2}{*}{$\begin{array}{l}\text { Das } \\
\text { Tier } \\
\text { erhielt } \\
\text { pro die } \\
\mathrm{g} \mathrm{N}\end{array}$} & \multicolumn{4}{|c|}{ Es wurden ausgeschieden $\mathbf{g} \mathbf{N}$} & \multicolumn{2}{|c|}{ Also angesetzt $\mathrm{N}$} & \multirow{2}{*}{$\begin{array}{c}\text { Ge- } \\
\text { wicht } \\
\text { des } \\
\text { Tieres } \\
\text { kg }\end{array}$} \\
\hline & & $\begin{array}{c}\text { im } \\
\text { Harn }\end{array}$ & $\begin{array}{l}\text { im } \\
\text { Kot }\end{array}$ & $\begin{array}{l}\text { in den Epi- } \\
\text { dermis- } \\
\text { gebilden }\end{array}$ & Sa. & g & $\begin{array}{l}\% \text { der } \\
\text { Ein- } \\
\text { nahme }\end{array}$ & \\
\hline 27./28. V. & 3,42 & 3,24 & 0,63 & 0,08 & 3,95 & $-0,53$ & $-15,50$ & 7,520 \\
\hline $28 . / 29$. & 3,42 & 2,99 & 0,63 & 0,08 & 3,70 & $-0,28$ & $-8,19$ & - \\
\hline $29 . / 30$. & 3,42 & 2,87 & 0,63 & 0,08 & 3,58 & $-0,16$ & $-4,68$ & - \\
\hline 30./31. & 3,42 & 3,06 & 0,63 & 0,08 & 3,77 & $-0,35$ & $-10,23$ & - \\
\hline 31./1. VI. & 3,42 & 2,90 & 0,63 & 0,08 & 3,61 & $-0,19$ & $-5,56$ & 7,560 \\
\hline Summa & 17,10 & 15,06 & $3,1 \check{5}$ & 0,40 & 18,61 & $-1,51$ & $|-8,83|$ & - \\
\hline \multicolumn{9}{|c|}{ Also im Mittel von 5 Tagen: } \\
\hline & 3,42 & 3,01 & 0,63 & 0,08 & 3,72 & $-0,30 \mid$ & $|-8,83|$ & - \\
\hline
\end{tabular}

Die Nahrung war also unzureichend, da das Tier täglich $0,3 \mathrm{~g} \mathrm{~N}$ von seinem Körperbestande einbüßte.

Ammonacetatperiode (1.-6. Juni 1907).

Das Tier Nr. 2 (wachsende Hündin) erhielt pro die:

$$
\begin{aligned}
& 80,00 \mathrm{~g} \text { Fleisch } \quad=2,44 \mathrm{~g} \mathrm{~N} \text { und } 150,48 \mathrm{Kal} \text {. } \\
& 80,00 \text {, Reis }=0,86 \text { > , 302,96 , } \\
& \text { 5,79, Ammonacetat }=1,00, \text {, , 20,72, }
\end{aligned}
$$

\begin{tabular}{|c|c|c|c|c|c|c|c|c|}
\hline \multirow[b]{2}{*}{$\begin{array}{c}\text { Datum } \\
1907\end{array}$} & \multirow{2}{*}{\begin{tabular}{|c|} 
Das \\
Tier \\
erhielt \\
pro die \\
g N
\end{tabular}} & \multicolumn{4}{|c|}{ Es wurden ausgeschieden $\mathrm{g} \mathrm{N}$} & \multicolumn{2}{|c|}{ Also angesetzt $\mathrm{N}$} & \multirow{2}{*}{\begin{tabular}{|c} 
Ge- \\
wicht \\
des \\
Tieres \\
kg
\end{tabular}} \\
\hline & & $\begin{array}{c}\text { im } \\
\text { Harn }\end{array}$ & $\begin{array}{l}\text { im } \\
\text { Kot }\end{array}$ & $\begin{array}{c}\text { in den Epi- } \\
\text { dermis- } \\
\text { gebilden }\end{array}$ & Sa. & $\mathbf{g}$ & $\begin{array}{l}\% \text { der } \\
\text { Ein- } \\
\text { nahme }\end{array}$ & \\
\hline 1.j2. VI. & 4,42 & 3,71 & 0,43 & 0,08 & 4,22 & 0,20 & 4,52 & 7,560 \\
\hline $2 . / 3$. & 4,42 & 53 & 0,43 & 0,08 & 4,04 & 0,38 & 8,60 & - \\
\hline 3.14 . & 4,42 & 3,49 & 0,43 & 0,08 & 4,00 & 0,42 & 9,50 & - \\
\hline 4.15 . & 4,42 & 3,53 & 0,43 & 0,08 & 4,04 & 0,38 & 8,60 & - \\
\hline $5 . / 6$. & 4,42 & 3,68 & 0,43 & 0,08 & 4,19 & 0,23 & 5,20 & 7,700 \\
\hline & 22,10 & $\overline{17,94}$ & 2,15 & 0,40 & 20,49 & 1,61 & - & - \\
\hline \multicolumn{9}{|c|}{ Also im Mittel von 5 Tagen: } \\
\hline & 4,42 & 3,59 & 0,43 & 0,08 & 4,10 & 0,32 & 7,29 & - \\
\hline
\end{tabular}

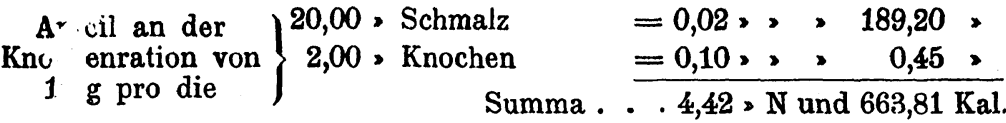

N-Bilanzen:

Hoppe-Seyler's Zeitschrift f. physiol. Chemie. LXXIX. 
Abschließende Grundrationsperiode (6.-13. Juni 1907).

Das Tier Nr. 2 (wachsende Hündin) erhielt pro die:

$$
\begin{aligned}
& 80,00 \mathrm{~g} \text { Fleisch }=2,44 \mathrm{~g} \mathrm{~N} \text { und } 150,48 \mathrm{Kal} \text {. } \\
& 80,00 \gg \text { Reis }=0,86 \gg \gg 302,96 \text {, } \\
& \text { Anteil an der Knochen- } 20,00 » \text { Schmalz }=0,02 » \gg \text { 189,20 》 }
\end{aligned}
$$$$
\text { ration von } 14 \mathrm{~g} \text { pro die }\} 2,00 \otimes K n o c h e n=0,10 \gg \otimes 0,45 \text { ॰ }
$$

\begin{tabular}{|c|c|c|c|c|c|c|c|c|}
\hline \multirow[b]{2}{*}{$\begin{array}{c}\text { Datum } \\
1907 \\
\end{array}$} & \multirow{2}{*}{$\left|\begin{array}{c}\text { Das } \\
\text { Tier } \\
\text { erhielt } \\
\text { pro die } \\
\text { g N }\end{array}\right|$} & \multicolumn{4}{|c|}{ Es wurden ausgeschieden $\mathrm{g} \mathrm{N}$} & \multicolumn{2}{|c|}{ Also angesetzt N } & \multirow{2}{*}{$\begin{array}{c}\text { Ge- } \\
\text { wicht } \\
\text { des } \\
\text { Tieres } \\
\text { kg }\end{array}$} \\
\hline & & $\begin{array}{l}\text { im } \\
\text { Harn }\end{array}$ & $\begin{array}{l}\text { im } \\
\text { Kot }\end{array}$ & $\mid \begin{array}{c}\text { in den Epi- } \\
\text { dermis- } \\
\text { gebilden }\end{array}$ & Sa. & g & $\begin{array}{c}\% \text { der } \\
\text { Ein- } \\
\text { nahme }\end{array}$ & \\
\hline $6 . / 7$. VI. & 3,42 & 2,83 & 0,48 & 0,07 & 3,38 & 0,04 & 1,16 & 7,700 \\
\hline $7 . / 8$. & 3,42 & 2,70 & 0,49 & 0,08 & 3,27 & 0,15 & 4,39 & - \\
\hline 8./9. & 3,42 & 2,76 & 0,49 & 0,08 & 3,33 & 0,09 & 2,63 & - \\
\hline $9 . / 10$. & 3,42 & 2,77 & 0,49 & 0,08 & 3,34 & 0,08 & 2,34 & - \\
\hline $10 . / 11$. & 3,42 & 2,77 & 0,49 & 0,08 & 3,34 & 0,08 & 2,34 & - \\
\hline 11./12. & 3,42 & 2,87 & 0,49 & 0,08 & 3,44 & $-0,02$ & $-0,58$ & - \\
\hline $12 . / 13$. & 3,42 & 2,85 & 0,49 & 0,08 & 3,42 & 0 & 0 & 7,740 \\
\hline imma & $\overline{23,94}$ & 19,55 & 3,42 & 0,55 & $\overline{23,52}$ & - & - & - \\
\hline
\end{tabular}

Summa . . 3,42 g N und 643,09 Kal.

N-Bilanzen:

Also im Mittel von 7 Tagen:

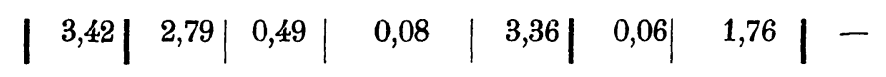

Wir ersehen aus den vorstehenden Zahlen für die 3 Perioden, daß durch die Zulage von $1 \mathrm{~g}$ Stickstoff in Form von Ammonacetat im Vergleich zur Vorperiode $0,62 \mathrm{~g} \mathrm{~N}$, im Vergleich zur Nachperiode $0,26 \mathrm{~g} \mathrm{~N}$ und im Vergleich zu dem Durchschnittswert aus beiden Perioden 0,44 $\mathrm{g} \mathrm{N}$, entsprechend $44 \%$ des Ammonacetatstickstoffes täglich von dem Tier retiniert wurden.

Handelt es sich bei dieser N-Retention von täglich $0,4 \mathrm{~g}$ um eine direkte Verwertung des Ammonacetatstickstoffes durch die tierischen Zellen, oder lassen die Befunde andere Deutungen zu? Wir wollen einmal annehmen, daß es sich hier um eine indirekte Wirkung handelte, daß also ähnlich, wie nach Kohlenhydrat- oder Fettzufuhr, eine sogenannte eiweißsparende Wirkung durch die Ammonacetatgabe erzielt worden wäre. Unter dieser Voraussetzung ergibt sich folgendes: 
Der Energiegehalt des verabreichten Ammonacetates betrug 20,72 Kal., von denen, falls der gesamte Ammonacetatstickstoff als Harnstoff zur Ausscheidung gelangt wäre, 5,45 Kal. ungenutzt den Körper verlassen hätten, es wären somit noch $20,72-5,45=15,27 \mathrm{Kal}$. des Ammonacetates für den Organismus verfügbar gewesen. Es gibt keine N-freien Nährstoffe, von denen isodyname Mengen, also 15,27, oder auch 20,72 Kal., eine Retention von 0,4 g Stickstoff bewirken könnten. Es muß also der Ammonacetatstickstoff bei dem vorliegenden Versuch direkt verwertet worden sein, sofern eine nachträgliche Ausscheidung nicht stattgefunden hat. Wenn wir uns hierauf die betreffenden Daten über den N-Gehalt des Harnes der abschließenden Grundfutterperiode an den einzelnen Tagen ansehen, so erkennen wir, daß von einer nachträglichen Ausscheidung des verabreichten Ammonacetatstickstoffes nicht die Rede ist. Ein etwaiger Einwand, daß der Ammonacetatstickstoff noch später als nach 7 Tagen zur Ausscheidung gelangen könnte, wäre leicht zu widerlegen; denn erstens hätte die Ausscheidung schon an den ersten Tagen der abschließenden Grundfutterperiode wenigstens begonnen haben müssen und zweitens wurden an demselben Tier ohne Unterbrechung weitere Versuche durchgeführt, in denen sich eine nachträgliche vermehrte $\mathrm{N}$-Ausscheidung unbedingt gezeigt hätte, was jedoch nicht der Fall war (siehe die Originalarbeit, 1. c., S. 1, Anm. 3). Beim Ammonacetat handelt es sich zudem um einen im tierischen Organismus leicht zersetzlichen Stoff, dessen Stickstoff, sofern die Bedingungen für eine Retention, z. B. infolge reichlicher Nahrung, bezw. der Tendenz des Organismus, das N-Gleichgewicht zu erreichen, ungünstig sind, sehr schnell im Urin wiedererscheint (siehe hierzu die auf S. 1, Anm. 2 zitierte Arbeit). Es war durch meine mitgeteilten Versuche somit erwiesen, daß der Ammoniakstickstoff auch vom Fleischfresser verwertet $z u$ werden vermag.

Außer für das Ammonacetat habe ich an der wachsenden Hündin und mein Mitarbeiter Yakuwa an einer zweiten Hündin unter analogen Versuchsbedingungen in Übereinstimmung gefunden, daß $23 \%$ des Acetamidstickstoffes und ebenfalls ein ziemlich hoher Prozentsatz vom Stickstoff des Amidgemisches retiniert wurden.

Nun behaupten Grafe und Schläpfer irrtümlich, ich hätte als Ursache für die N-Retentionen die Tätigkeit der Darmbakterien angesehen. Demgegenüber stelle ich fest, aus den in Betracht kommenden Befunden gefolgert zu haben, daß der Fleischfresser seine N-Bilanzen aus den Amidsubstanzen günstiger zu gestalten vermag. Ich habe, lange bevor Grafe und Schläpfer ihre Versuche in Angriff nahmen, mich mehrfach dahin geäußert, daß im Hinblick auf die im Verdauungstraktus beim Karnivoren im Vergleich zum Herbivoren sehr zurücktretende Bakterientätigkeit zu folgern wäre, daß die tierische Zelle so einfache $\mathrm{N}$-haltige Verbindungen, wie das Ammonacetat direkt verwerten, ja dieselben vielleicht 
sogar zu höher konstituierten N-haltigen Stoffen aufbauen kann. ") $\left.{ }^{2}\right)$ Diese Deutung ist durch die Forschungen von Knoop ${ }^{3}$ ) und $\mathrm{Embden}^{4}$ ) sehr wahrscheinlich geworden.

Die Folgerungen von Grafe und Schläpfer aus ihrer Arbeit sind also nur eine Bestätigung meiner früheren diesbezüglichen Resultate.

1) W. Völtz, Über die Bedeutung der Amidsubstanzen für die tierische Ernährung. Landwirtschaftliche Jahrbücher, Festschrift zur Feier des 70. Geburtstages von H. Thiel, S. 433-448, 1909.

S. 668 .

2) W. Völtz, Jahresbericht für Tierchemie über das Jahr 1910,

s) Knoop, Über den physiologischen Abbau der Säuren und die Synthese einer Aminosäure im Tierkörper. Diese Zeitschrift, Bd. 67, S. 489, 1910.

4) Embden, Über synthetische Bildung von Aminosäuren in der Leber. Biochemische Zeitschrift, Bd. 29, S. 423, 1910. 